\title{
Electrolytic treatment characteristics of boron-doped nanocrystalline diamond/amorphous carbon composite films prepared by coaxial arc plasma deposition
}

\author{
Takumi Takenaga ${ }^{\mathrm{a}}$, Masaya Onishi ${ }^{\mathrm{a}}$, Daisuke Fujimoto ${ }^{\mathrm{a}}$, Yoshihiro Nojiri ${ }^{\mathrm{b}}$, and Takeshi Hara ${ }^{\mathrm{a}}{ }^{*}$ \\ ${ }^{a}$ Department of Creative Engineering, National Institute of Technology, \\ Ariake College, Omuta, Fukuoka 836-8585, Japan \\ ${ }^{\mathrm{b}}$ Department of Chemical and Biological Engineering, National Institute of Technology, \\ Sasebo College, Sasebo, Nagasaki 857-1193, Japan \\ *Corresponding Author: hara@ariake-nct.ac.jp
}

\begin{abstract}
Boron-doped nanocrystalline diamond/amorphous carbon composite (NCD/a-C) films were fabricated by coaxial arc plasma deposition (CAPD). Electrical conductivity measurements and thermal analysis confirmed that the deposited films exhibited p-type semiconductor behavior. Cyclic voltammetry measurements of deposited films showed a wide electrical potential window and an extremely low background current. 4-Nitrophenol (4-NP) aqueous solution, which is a recalcitrant substance, was decomposed via electrolytic treatment using a batch-type electrolytic treatment system wherein the B-doped NCD/a-C films were used as anodes. UV-vis spectra showed that the absorbance of 4-NP at $400 \mathrm{~nm}$ decreased with increasing electrolytic treatment time. This behavior implies that the B-doped NCD/a-C films deposited by CAPD are potential electrode materials for use in the electrolytic degradation of recalcitrant substances.
\end{abstract}

Keywords: nanocrystalline diamond, coaxial arc plasma deposition, electrolytic treatment.

\section{Introduction}

Boron-doped diamond (BDD) films have attracted much attention as electrode materials for wastewater treatment $^{(1,2)}$ because of their superior chemical characteristics, which include a wide potential window, extremely low background current, and high chemical stability in various solutions. Industrial effluents contain some persistent substances, typified by phenolic pollutants.
These substances are difficult to decompose using conventional noble-metal electrodes for electrochemical treatment, i.e., Pt electrodes. In cases where such electrodes are used for electrochemical treatment, electrolysis of water preferentially occurs because of the electrodes' narrow potential window, which is much narrower than that of BDD electrodes. Aqueous waste containing various persistent substances is reported to be decomposed by BDD films used as anode electrodes ${ }^{(3)}$. BDD electrodes have also been used as electrodes for electrosynthesis ${ }^{(4)}$ and electrochemical sensors ${ }^{(5)}$; thus, BDD is an electrode material with numerous potential electrochemical applications.

Diamond films are usually fabricated by chemical vapor deposition (CVD); they are promising for various engineering applications as electrochemical, electronic device, and hard coating materials ${ }^{(6,7)}$. However, CVD of diamond films ${ }^{(8,9)}$ requires (i) a general substrate temperature of approximately $800^{\circ} \mathrm{C}$ or higher, (ii) pretreatment of substrates using diamond powder, which is called seeding, and (iii) the presence of molecular hydrogen. If these requirements could be circumvented, then the industrial application of diamond films in various fields would be further accelerated.

Nanocrystalline diamond/hydrogenated amorphous carbon composite (NCD/a-C:H) films and $\mathrm{NCD}$ /nonhydrogenated amorphous carbon composite (NCD/a-C) films comprising diamond crystallites in matrices of a-C:H and a-C have attracted attention because of their unique characteristics, which include a low coefficient of friction, high thermal stability, and large 
optical absorption coefficient ${ }^{(10-13)}$. Diamond crystallites with diameters less than $10 \mathrm{~nm}$ are called ultrananocrystalline diamond (UNCD). UNCD/a-C, $\mathrm{UNCD} / \mathrm{a}-\mathrm{C}: \mathrm{H}, \quad \mathrm{NCD} / \mathrm{a}-\mathrm{C}$, and $\mathrm{NCD} / \mathrm{a}-\mathrm{C}: \mathrm{H}$ films are expected to have extensive industrial applications ${ }^{(14,15)}$. The superior mechanical, physical, and chemical characteristics of these films are assumed to be a consequence of NCDs and UNCDs in an a-C or a-C:H matrix existing as diamond crystallites. Such films are fabricated by $\mathrm{CVD}^{(10,12-14)}$. By contrast, reports of the fabrication of such films by physical vapor deposition (PVD) methods are scarce.

Here we used coaxial arc plasma deposition (CAPD), which is a PVD method, to grow UNCD/a-C and NCD/a-C films; we successfully fabricated UNCD/a-C films with diamond crystallite diameters of approximately $1.5 \mathrm{~nm}$ at a substrate temperature of room temperature under vacuum using our previously reported method ${ }^{(16)}$. Moreover, we did not pretreat the substrate with diamond powder because the nucleation of diamond occurs autonomously in this method.

Using a combination of in situ emission spectroscopy and time-resolved spectroscopy, Hanada et al. confirmed the presence of $\mathrm{C}^{+}$ions with a high density of energetic species in the plasma used in the CAPD process ${ }^{(17)}$. A previous report of diamond nucleation induced by the bombardment of highly energetic $\mathrm{C}^{+}$ions onto an a-C matrix ${ }^{(18)}$ suggests that the impact of such a high density of energetic carbon species at the substrate leads to a supersaturated condition for the growth of the diamond crystallites. As such, the growth of diamond crystallites by CAPD in vacuum may be possible even when the substrate is at room temperature. Although the literature contains substantially fewer reports concerning these films compared to the number of reports concerning films prepared by CVD, the development of diamond crystalline CAPD films for various industrial applications such as electronic devices ${ }^{(19,20)}$ and coatings ${ }^{(11)}$ has been steadily progressing.

In our previous study, we grew B-doped UNCD/a-C films by CAPD at room temperature under vacuum and confirmed that these films exhibit two superior electrochemical characteristics similar to those of commercially available BDD electrodes: a wide potential window and an extremely low background current ${ }^{(21)}$. We used the deposited films to decompose aqueous solutions of ethanol, and the electrolytic performance of the BDD electrodes greatly exceeded the capability of Pt electrodes; in fact, their performance was comparable to that of commercial BDD electrodes $^{(21)}$.
Here we attempted to decompose 4-nitrophenol (4-NP) aqueous solutions using B-doped NCD/a-C films. 4-NP is one of the persistent substances in industrial effluents. Although the literature contains some examples of 4-NP decomposition using BDD electrodes prepared by $\mathrm{CVD}^{(1,2)}$, the use of B-doped $\mathrm{NCD} / \mathrm{a}-\mathrm{C}$ films prepared by $\mathrm{CAPD}$ methods has not been previously reported. As previously discussed, the CAPD method offers unique advantages for the formation of diamond crystallites: (i) diamond crystallites can be grown on a substrate at room temperature; (ii) pretreatment of the substrate with diamond powder is not necessary for diamond growth; and (iii) an ambient hydrogen gas is not required for the growth of diamond crystallites. Diamond crystallites cannot be grown under these conditions using CVD, irrespective of the diamond crystallites' size. If the electrolytic treatment performance of $\mathrm{B}$-doped $\mathrm{NCD} / \mathrm{a}-\mathrm{C}$ films prepared by CAPD is similar to that of BDD electrodes, then they will be attractive as new high-performance electrodes for electrolytic treatment.

\section{Experimental Procedure}

Figure 1 shows a schematic of the film deposition apparatus. B-doped NCD/a-C films with a thickness of approximately $1.4 \mu \mathrm{m}$ were deposited by CAPD under a vacuum better than $10^{-3} \mathrm{~Pa}$ onto low-resistivity p-type $\mathrm{Si}$ (100) substrates; the substrate temperature was maintained at room temperature, and a graphite target containing 5 at.\% $\mathrm{B}$ was used. The distance between the substrate and the target was $20 \mathrm{~mm}$. An arc voltage of $100 \mathrm{~V}$ was applied to an arc plasma gun equipped with a $720 \mu \mathrm{F}$ capacitor. The repetition rate of pulsed discharges was $5 \mathrm{~Hz}$. The growth of the NCD crystallites was investigated by X-ray diffraction (XRD) using a diffractometer (Rigaku Ultima

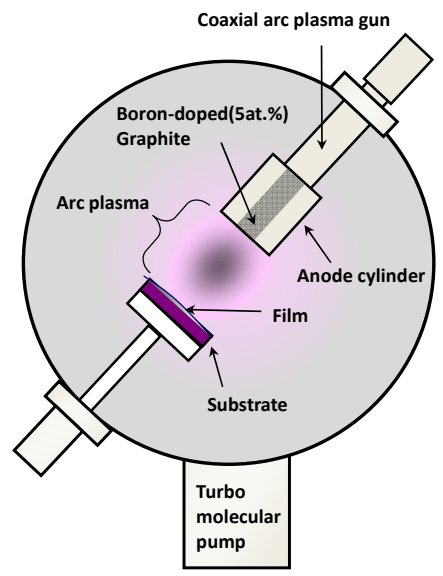

Fig. 1. Schematic of the film deposition apparatus. 
IV/SN) equipped with a $\mathrm{Cu}-\mathrm{K} \alpha$ radiation source and semiconductor counter detectors. Raman spectroscopy (JASCO Corporation NRS-2000) was used to study the film structure.

The potential window and background current were measured by cyclic voltammetry (CV) with the electrodes immersed in an electrolytic solution of aqueous $1.0 \mathrm{~mol} / \mathrm{L}$ $\mathrm{H}_{2} \mathrm{SO}_{4}$. The working electrodes, the secondary electrode, and the reference electrode were the deposited films, Pt, and an $\mathrm{Ag} / \mathrm{AgCl}$ electrode, respectively.

The electrolytic treatment of 4-NP aqueous solutions was conducted using a batch-type electrolytic system with the deposited films used as the anode and a stainless steel electrode used as the cathode. The distance between these electrodes was $5.0 \mathrm{~mm}$. The concentration of the $\mathrm{Na}_{2} \mathrm{SO}_{4}$ electrolytic solution was $0.20 \mathrm{~mol} / \mathrm{L}$. Twenty-five milliliters of a 4-NP aqueous solution of $100 \mathrm{mgTOC}$ (total organic carbon) /L was prepared and run through upward flow at $0.60 \mathrm{~L} / \mathrm{h}$. The current density was $1.5 \mathrm{~A} / \mathrm{dm}^{2}$. The total electrolytic treatment time was $60 \mathrm{~min}$. One milliliter of the electrolytically treated solution was picked up by syringe every $20 \mathrm{~min}$ and mixed with $1.0 \mathrm{~mol} / \mathrm{L}$ carbonate buffer solution $\left(\mathrm{Na}_{2} \mathrm{CO}_{3}+\mathrm{NaHCO}_{3}\right)$ adjusted to $\mathrm{pH}$ 9.8; the absorbance of the mixed solution was measured using a UV-vis spectrophotometer (Shimadzu UV-2550PC).

\section{Results and Discussion}

Figure 2 shows the XRD pattern of the deposited film. A semiconductor counter detector was used to improve the detection sensitivity. Only an extremely weak peak at $44.28^{\circ}$ due to diamond (111) was observed. The diamond (220) diffraction peak at $75.97^{\circ}$, which is approximately five times weaker than that of diamond (111), was not observed; it was obscured by the background data. We proposed this measurement technique of using a semiconductor detector in our previous report ${ }^{(16)}$; it is a simple method for detecting NCD and UNCD in an a-C matrix. Furthermore, this method can be used to characterize films. Although NCD and UNCD in an a-C matrix have often been characterized using powder XRD in conjunction with a synchrotron radiation source ${ }^{(17,19,20)}$, this technique is not readily available in laboratories.

We used the Scherrer equation to calculate diamond crystallite diameters of approximately $40 \mathrm{~nm}$ on the basis of the diamond (111) peak in Fig. 2. In our previous study, we grew UNCD with crystallite diameters of $1.5 \mathrm{~nm}$ in an a-C matrix by $\mathrm{CAPD}^{(16)}$; by contrast, the diameter of the

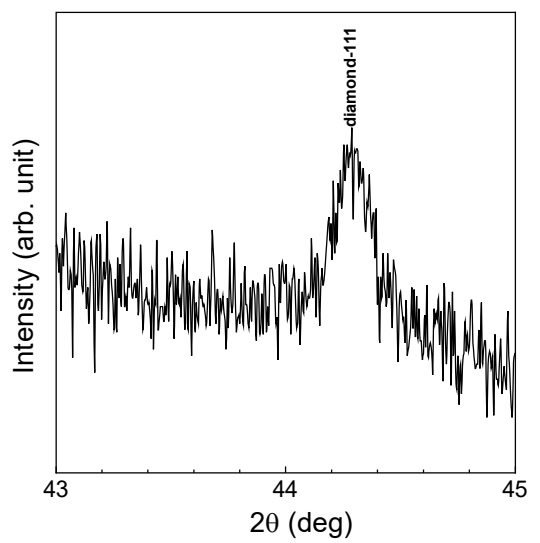

Fig. 2. XRD pattern of a deposited NCD film with detection using a semiconductor counter detector.

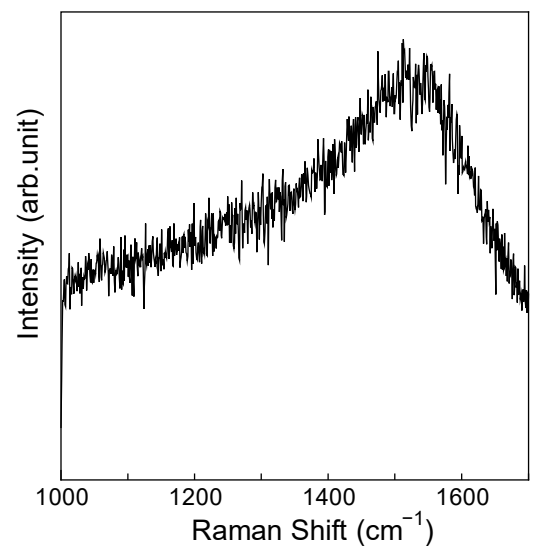

Fig. 3. Typical Raman spectra of B-doped NCD/a-C films prepared by CAPD.

diamond crystallites herein is approximately 27 times greater than that of the undoped UNCD/a-C films. Katamune et al. reported that the grain size of UNCD prepared by CAPD increases with increasing B content in this method ${ }^{(22)}$. In their study, the grain size of the deposited UNCD with a B concentration of 10 at.\% was 40 times greater than that of the undoped films. They attributed the dramatically enhanced growth to the dilation of diamond lattices by the incorporation of $\mathrm{B}$ atoms into the lattices ${ }^{(22)}$. Our study corroborates their results.

A typical Raman spectrum of the deposited B-doped $\mathrm{NCD} / \mathrm{a}-\mathrm{C}$ films is shown in Fig. 3. A broad G-band is observed at approximately $1580 \mathrm{~cm}^{-1}$; this peak is typically observed in the Raman spectra of a-C films. We did not observe a broad, weak peak at $1150 \mathrm{~cm}^{-1}$; this peak is often observed in the spectra of NCD/a-C:H and UNCD/a-C:H films and originates from trans-polyacetylene segments at the grain boundaries of NCD grains and UNCD grain surfaces ${ }^{(23)}$. However, our films contained no $\mathrm{CH}_{x}$ bonds due to trans-polyacetylene because we deposited the films 


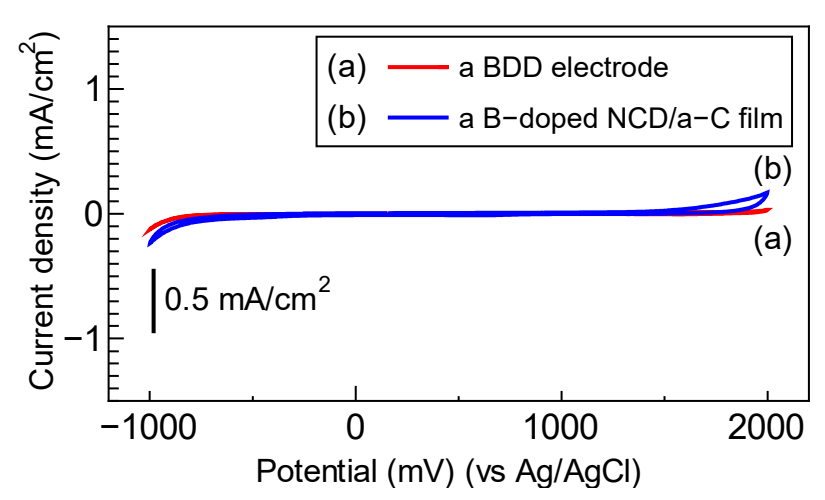

Fig. 4. Cyclic voltammograms of (a) a BDD electrode and (b) a B-doped NCD/a-C film.

under vacuum, without a hydrogen atmosphere. The XRD and Raman analyses confirmed that we prepared B-doped NCD/a-C films by CAPD. Moreover, the electrical conductivity of the films increased with increasing temperature in the range from 100 to $500 \mathrm{~K}$ and thermoelectric force value measurements indicated that the films exhibit p-type conduction. Thus, the deposited films exhibit p-type semiconducting characteristics.

We used CV to evaluate the films' potential window and background current, which are fundamental electrochemical characteristics. The results are similar to those for BDD electrodes, which exhibit a wide potential window and an extremely low background current, as shown in Fig. 4. This result implies that the B-doped NCD/a-C films prepared by CAPD have strong potential for use as electrolytic treatment electrodes.

4-NP aqueous solutions of $100 \mathrm{mgTOC} / \mathrm{L}$ were used in electrolytic treatment experiments with a BDD electrode, the B-doped NCD/a-C films, and Pt electrodes in a batch-type electrolytic system. The BDD and Pt electrodes were commercially produced. We observed that the intensity of the characteristic peak of 4-NP at $400 \mathrm{~nm}$ in the UV-vis spectra decreased with increasing electrolytic treatment time when these electrodes were used as the anode. The BDD electrodes demonstrated particularly strong electrolytic treatment characteristics, and the rate of 4-NP absorbance reduction was approximately $36 \%$ after 60 min, as shown in Fig. 5(a). B-doped NCD/a-C films also exhibited good 4-NP degradation capability in aqueous solutions, as shown in Fig. 5(b). The decreasing trend of the absorbance at $400 \mathrm{~nm}$ with increasing electrolytic treatment time was similar to that observed for BDD electrodes. The reduction rate of the characteristic peak associated with 4-NP was approximately $31 \%$ after $60 \mathrm{~min}$. Although the degradation capability of the B-doped NCD/a-C films
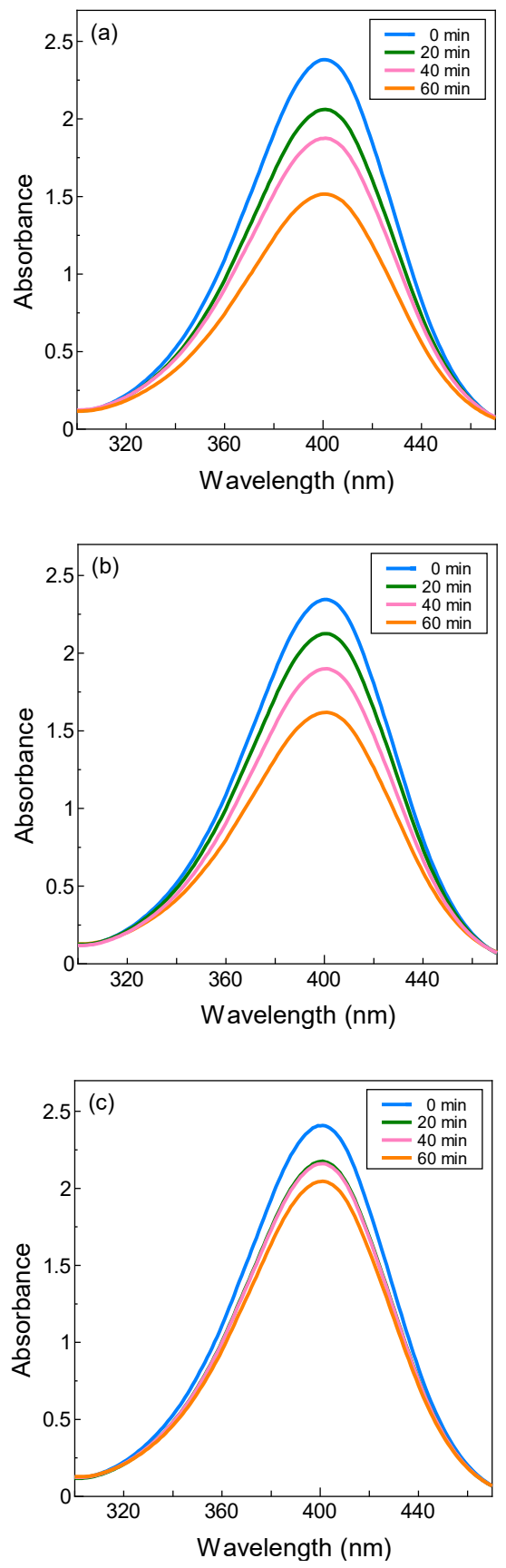

Fig. 5. UV-vis absorption spectra for the reduction of 4-NP for $60 \mathrm{~min}$ via electrolytic treatment using (a) a BDD electrode, (b) a B-doped NCD/a-C film, and (c) a Pt electrode as the anode.

prepared by CAPD was slightly worse than that of the BDD electrodes, the results nonetheless indicate that the B-doped NCD/a-C films prepared by CAPD, like the BDD electrodes, are strong candidate electrodes for the electrolytic treatment of effluents. By contrast, the reduction rate of the $\mathrm{Pt}$ electrodes at $60 \mathrm{~min}$ was approximately $14 \%$, as estimated from Fig. 5(c). This value is less than half the reduction rates exhibited by the BDD 
and B-doped NCD/a-C film electrodes.

We compared the performance of the BDD electrodes with that of the B-doped NCD/a-C films. The electrode areas of all of the electrodes are the same at approximately $15 \mathrm{~mm}^{2}$. The BDD electrodes exhibit a single-phase polycrystalline structure, whereas the deposited films comprise an NCD/a-C composite. In the electrode area, the densities of diamond crystallites fundamentally differ from those of B-doped NCD/a-C films. The electrolytic treatment performance should improve if the growth of NCD in the deposited films is enhanced. However, the growth of B-doped NCD/a-C films by CAPD has some unique conditions for film deposition and these conditions are not suitable for growing diamond crystallites by CVD. We posit that these unique conditions are more attractive than the performance difference between BDD electrodes for industrial applications and B-doped NCD/a-C prepared by CAPD as new electrode materials.

\section{Conclusions}

The B-doped NCD/a-C films prepared by CAPD decomposed 4-NP, and their electrolytic treatment performance was superior to that of Pt electrodes. Their performance at $60 \mathrm{~min}$ was slightly inferior to that of BDD electrodes. However, the formation conditions of the electrodes were much more straightforward than the conditions required for CVD. Thus, B-doped NCD/a-C films prepared by CAPD offer great advantages for industrial application as electrodes for electrolytic treatment.

\section{Acknowledgment}

This study was supported by Kurita Water and Environment Foundation. XRD measurements were performed at the Department of Chemical and Biological Engineering, National Institute of Technology, Sasebo College. Raman spectrum equipment was done at the Center for Advanced Instrument Analysis, Kyushu University.

\section{References}

(1) M.A. García-Morales, G. Roa-Morales, C. Barrera-Díaz, B. Bilyeu, M.A. Rodrigo: "Synergy of electrochemical oxidation using boron-doped diamond
(BDD) electrodes," Electrochemistry Communications, Vol. 27, pp. 34-37, 2013.

(2) A. Morãoa, A. Lopes, M.T. Pessoa de Amorimb, I.C. Gonçalves: "Degradation of mixtures of phenols using boron doped diamond electrodes for wastewater treatment," Electrochimica Acta, Vol. 49, pp. 1587-1595, 2004.

(3) P. Cañizares, C. Sáez, J. Lobato, M.A. Rodrigo: "Electrochemical treatment of 4-nitrophenol-containing aqueous wastes using boron-doped diamond anodes," Industrial \& Engineering Chemistry Research, Vol. 43, No. 9, pp. 1944-1951, 2004.

(4) S.R. Waldvogel, B. Elsler: "Electrochemical synthesis on boron-doped diamond," Electrochimica Acta, Vol. 82, pp. 434-443, 2012.

(5) T. Kondo, K. Sakai, T. Watanabe, Y. Einaga, M. Yuasa: "Electrochemical detection of lipophilic antioxidants with high sensitivity at boron-doped diamond electrode," Electrochimica Acta, Vol. 95, pp. 205-211, 2013.

(6) K. Ueda, M. Kasu, Y. Yamauchi, T. Makimoto, M. Schwitters, D.J. Twitchen, G.A. Scarsbrook, S.E. Coe: "Diamond FET using high-quality polycrystalline diamond with $f_{T}$ of $45 \mathrm{GHz}$ and $f_{\max }$ of $120 \mathrm{GHz}$," IEEE Electron Device Letters, Vol. 27, No. 7, pp. 570-572, 2006.

(7) F.J.G. Silva, A.J.S. Fernandes, F.M. Costa, A.P.M. Baptista, E. Pereira: "A new interlayer approach for CVD diamond coating of steel substrates," Diamond and Related Materials, Vol. 13, pp. 823-833, 2004.

(8) X. Pei, S. Cheng, Y. Ma, D. Wu, J. Liu, Q. Wang, Y. Yang, H. Li: "Structure and wettability property of the growth and nucleation surfaces of thermally treated freestanding CVD diamond films," Applied Surface Science, Vol. 346, pp. 189-193, 2015.

(9) M. Zhang, B. Gu, L. Wang, Y. Xia: "Preparation and characterization of (100)-textured diamond films obtained by hot-filament CVD," Vacuum, Vol. 75, pp. 84-89, 2005.

(10) M. Mohr, L. Daccache, S. Horvat, K. Brühne, T. Jacob: "Influence of grain boundaries on elasticity and thermal conductivity of nanocrystalline diamond films,' Acta Materialia, Vol. 122, pp. 92-98, 2017.

(11)H. Naragino, M. Egiza, A. Tominaga, K. Murasawa, H. Gonda, M. Sakurai, T. Yoshitake: "Room-temperature hard coating of ultrananocrystalline diamond/nonhydrogenated amorphous carbon 
composite films on tungsten carbide by coaxial arc plasma deposition," Japanese Journal of Applied Physics, Vol. 55, No. 3, pp. 0303021-0303024, 2016.

(12) M. Sobaszek, Ł. Skowronski, R. Bogdanowicz, K. Siuzdak, A. Cirocka, P. Zieba, M. Gnyba: "Optical and electrical properties of ultrathin transparent nanocrystalline boron-doped diamond electrodes," Optical Materials, Vol. 42, pp. 24-23, 2015.

(13) J. Anaya, S. Rossi, M. Alomari, E. Kohn, L. Tóth, B. Pécz, K.D. Hobart, T.J. Anderson, T.I. Feygelson, B.B. Pate: "Control of the in-plane thermal conductivity of ultra-thin nanocrystalline diamond films through the grain and grain boundary properties," Acta Materialia, Vol. 133, pp. 141-152, 2016.

(14) O.A. Williams, M. Daenen, J. D'Haen, K. Haenen, J. Maes, V.V. Moshchalkov, M. Nesládek, D.M. Gruen: "Comparison of the growth and properties of ultrananocrystalline diamond and nanocrystalline diamond," Diamond and Related Materials, Vol. 15, pp. 654-658, 2006.

(15) A. Zkria, Y. Katamune, T. Yoshitake: "Effects of Nitrogen doping on the electrical conductivity and optical absorption of ultrananocrystalline diamond/hydrogenated amorphous carbon films prepared by coaxial arc plasma deposition," Japanese Journal of Applied Physics, Vol. 55, No. 7S2, pp. 07LE011-07LE014, 2016.

(16) T. Hara, Y. Nojiri, K. Hanada, T. Yoshitake: "Detection methods of diamond diffraction peaks in ultrananocrystalline diamond/amorphous carbon composite films by X-ray diffraction measurement with semiconductor counter detector," Japanese Journal of Applied Physics, Vol. 54, No. 10, pp. 1080021-1080023, 2015.

(17) K. Hanada, T. Yoshitake, T. Nishiyama, K. Nagayama: "Time-resolved spectroscopic observation of deposition processes of ultrananocrystalline diamond/amorphous carbon composite films by using a coaxial arc plasma gun," Journal of Applied Physics, Vol. 49, No. 8, pp. 08JF091-08JF094, 2010.

(18) Y. Yao, M.Y. Liao, T. Köhler, T. Frauenheim, R.Q. Zhang, Z.G. Wang, Y. Lifshitz, S.T. Lee: "Diamond nucleation by energetic pure carbon bombardment," Physical Review B, Vol. 72, pp. 0354021-0354025, 2005.

(19) A. Zkria, Y. Katamune, T. Yoshitake: "Effects of Nitrogen doping on the electrical conductivity and optical absorption of ultrananocrystalline diamond/hydrogenated amorphous carbon films prepared by coaxial arc plasma deposition," Japanese Journal of Applied Physics, Vol. 55, No. 7S2, pp. 07LE011-07LE014, 2016.

(20)A. Zkria, H. Gima, M. Shaban, T. Yoshitake: "Electrical characteristics of nitrogen-doped ultrananocrystalline diamond/hydrogenated amorphous carbon composite films prepared by coaxial arc plasma deposition,” Applied Physics Express, Vol. 8, No. 9, pp. 095101-095103, 2015.

(21)T. Hara, M. Onishi, D. Fujimoto, T. Yoshitake: "Electrochemical characteristics of boron-doped ultrananocrystalline diamond/amorphous carbon composite films fabricated by coaxial arc plasma deposition," The Japanese Journal of the Institute of Industrial Applications Engineers [in Japanese], Vol. 4, No.1, pp. 31-32, 2016.

(22) Y. katamune, S. Ohmagari, T. Yoshitake: "Boron-induced dramatically enhanced grown of diamond grains in nanocrystalline diamond/hydrogenated amorphous carbon composite films deposited by coaxial arc plasma deposition," Japanese Journal of Applied Physics, Vol. 51, No. 7R, pp. 0780031-0780032, 2012.

(23) A.C. Ferrari and J. Robertson: "Origin of the $1150 \mathrm{~cm}^{-1}$ Raman mode in nanocrystalline diamond," Physical Review B, Vol. 63, pp. 1214051-1214054, 2001. 